\title{
Jefferson
}

Thomas Jefferson University

The Medicine Forum

Volume 3

Article 7

2001

\section{Melena with Fever}

\author{
Monica Patel \\ Thomas Jefferson University, monica.patel@jefferson.edu
}

Jennifer Wilhelm

Thomas Jefferson University

Follow this and additional works at: https://jdc.jefferson.edu/tmf

Part of the Endocrinology, Diabetes, and Metabolism Commons, and the Gastroenterology Commons Let us know how access to this document benefits you

\section{Recommended Citation}

Patel, Monica and Wilhelm, Jennifer (2001) "Melena with Fever," The Medicine Forum: Vol. 3 , Article 7.

DOI: https://doi.org/10.29046/TMF.003.009

Available at: https://jdc.jefferson.edu/tmf/vol3/iss1/7

This Article is brought to you for free and open access by the Jefferson Digital Commons. The Jefferson Digital Commons is a service of Thomas Jefferson University's Center for Teaching and Learning (CTL). The Commons is a showcase for Jefferson books and journals, peer-reviewed scholarly publications, unique historical collections from the University archives, and teaching tools. The Jefferson Digital Commons allows researchers and interested readers anywhere in the world to learn about and keep up to date with Jefferson scholarship. This article has been accepted for inclusion in The Medicine Forum by an authorized administrator of the Jefferson Digital Commons. For more information, please contact: JeffersonDigitalCommons@jefferson.edu. 


\section{Melena With Fever}

Monica Patel, MD, Resident Department of Internal Medicine 1999-2002

Jennifer Wilhelm, MD, Resident Department of Internal Medicine 1999-2002

The patient is a 62 year old Indian male with a past medical history significant for myasthenia gravis, hypertension, and diabetes mellitus, who presented to an outside hospital with fever, fecal incontinence, and melena. Work up at that hospital was remarkable for elevated liver function tests, heme-positive brown stool and a CT of the abdomen showing a thick ascending colon suggesting possible mass. The patient had an episode of desaturation and fever to 105 degrees Fahrenheit prior to transfer to Thomas Jefferson University Hospital for further evaluation. Upon arrival, the patient was in respiratory distress with oxygen saturation of $70 \%$ on 5 liters of oxygen by nasal cannula. He was immediately intubated.

The patient was a retired radiologist who emigrated from southern India 10 years ago. He has no history of tobacco, alcohol or illicit drug use. There were no known chemical exposures. His myasthenia gravis was diagnosed one year ago and was treated with pyridostigmine and prednisone. He had been on azathioprine which was discontinued three weeks prior to admission. At the outside hospital, the patient had been started on ampicillin/sulbactam for empiric coverage secondary to fever and possible infection; pyridostigmine had been discontinued.

Physical exam on admission revealed a temperature of 97.6 degrees Fahrenheit, respiratory rate of 40, regular pulse of $140 \mathrm{bpm}$, and blood pressure of $106 / 70 \mathrm{~mm} / \mathrm{Hg}$. Oxygen saturation was $100 \%$ on assist control rate of 16 breaths per minute, tidal volume $600 \mathrm{ml}, \mathrm{FiO} 250 \%$ and PEEP of +5 . In general, he was a well nourished, medium build Indian male, intubated and sedated. The patient had anicteric sclera, pink conjunctiva, no lymphadenopathy, no jugular venous distenstion, no thyromegaly. Heart rate was tachycardic with regular rhythm. Lung exam revealed coarse breath sounds bilaterally without wheezes. Abdomen was soft, non-tender, mildly distended without palpable mass or hepatosplenomegaly. Rectal exam revealed heme-positive brown stool. Extremities were warm with bilateral pitting edema.

Labwork revealed WBC 3,800/mL 3 (85\% neutrophils, $11 \%$ bands, $2 \%$ lymphocytes), hemoglobin 8.3 grams/dL, platelets $85,000 / \mathrm{mL}^{3}$. The patient had abnormal bicarbonate of $20 \mathrm{mmol} / \mathrm{L}$, elevated blood urea nitrogen (BUN) of $42 \mathrm{mg} / \mathrm{dL}$, high creatinine of $1.8 \mathrm{mg} / \mathrm{dL}$, and elevated glucose of $212 \mathrm{mg} / \mathrm{dL}$, His liver function tests were also abnormal with a low total protein of $4.1 \mathrm{~g} / \mathrm{dL}$, low albu$\mathrm{min}$ of $2.1 \mathrm{~g} / \mathrm{dL}$, and elevated alkaline phosphatase of 246 IU/L, AST of $47 \mathrm{IU} / \mathrm{L}$, ALT of $77 \mathrm{IU} / \mathrm{L}$, and normal total bilirubin $0.8 \mathrm{mg} / \mathrm{dL}$. Chest $\mathrm{x}$-ray showed mild pulmonary edema without infiltrate or mass.

The patient's pancytopenia was thought to be secondary to myasthenia gravis and he was started on IV immunoglobulin and hydrocortisone. Antibiotic coverage was expanded to piperacillin/tazobactam. Lower extremity dopplers revealed no venous thrombosis. CT of chest, abdomen, pelvis showed chronic wall thickening involving the cecum and portion of the ascending colon, abdominal ascites, bilateral pleural effusions and diffuse bilateral ground glass opacities within the lung fields (Figure 1). The patient underwent bone marrow biopsy for persistent pancytopenia. Results of the biopsy showed necrotizing granulomas positive for acid fast bacilli. Subsequently, a sputum culture revealed rare acid fast bacilli. MRI of the brain showed single focus of increased signal intensity in the left inferior frontal lobe with surrounding edema consistent with tuberculosis. The patient was diagnosed with disseminated tuberculosis and antituberculosis medications (ethambutol, pyrazinamide, rifampin, isoniazid) were started.

The patient soon developed melanotic stools. Creatinine began to rise shortly after intravenous dye load for CT scan. The patient required multiple transfusions of packed red blood cells, fresh frozen plasma, and platelets. The hospital course was further defined by worsening renal function requiring continuous veno-venous hemodialysis (CVVHD), continued melena without an identifiable source requiring frequent transfusions, and persistently elevated liver function tests despite discontinuation of rifampin. The patient continued to deteriorate clinically secondary to multiorgan failure. The family chose to withdraw care and the patient died shortly thereafter.

\section{Discussion}

Abdominal tuberculosis is an infrequent diagnosis especially in areas where tuberculosis is uncommon. The diagnosis is often missed because signs and symptoms are nonspecific with an insidious onset. Pathogenesis of 
abdominal tuberculosis includes swallowing expectorated sputum in the presence of active tuberculosis, hematogenous spread from pulmonary tuberculosis, ingestion of unpasteurized milk, or direct extension from adjacent organs. Common sites of abdominal involvement are the peritoneum, lymph nodes and ileocecal region. Pathologic changes involve inflammation of the submucosa and serosa which leads to edema, lymphatic hyperplasia, and eventually fibrosis. Mucosal ulceration results from endarteritis of submucosal vessels. Gross pathology is variable with areas of ulceration (either segmental or diffuse colitis), hypertrophy (polyps or mass), ulcerohypertrophic (mixed), or intestinal narrowing secondary to fibrous stricture or extraintestinal lymph node compression.

Clinical features are often nonspecific including abdominal pain, diarrhea, and weight loss. Concomitant active pulmonary tuberculosis is seen in only $15-20 \%$ with even less having a positive PPD test. Complications of abdominal tuberculosis include obstruction secondary to inflammatory thickening, fibrosis, or lymphadenopathy; perforation; fistula formation; bleeding; and malabsorption secondary to loss of mucosal integrity. The diagnosis of abdominal tuberculosis relies on maintaining a high index of suspicion within the appropriate patient population. Active pulmonary tuberculosis should lead the clinician to consider abdominal tuberculosis in the patient with concomitant abdominal symptoms.

Radiographic and endoscopic findings are also nonspecific often showing ileocecal involvement with mucosal ulceration, thickening of the ileocecal valve, strictures, or segmental narrowing. In order to make the definitive diagnosis, an endoscopy with biopsy or exploratory laparotomy is required. Biopsy will show caseating granulomas, with positive culture for acid fast rods.

A therapeutic trial of antitubercular medications may be appropriate when clinical suspicion is high. The drug regimens used for treating abdominal tuberculosis are those used for pulmonary tuberculosis. Treatment course ranges from 6 to 24 months depending on clinical course. Importance must be placed on maintaining nutrition. Surgical intervention is reserved for managing complications of obstruction, perforation, abscess or hemorrhage.

In summary, abdominal tuberculosis continues to be a diagnostic challenge as signs and symptoms are nonspecific and insidious in onset. Clinicians must maintain a high index of suspicion within the appropriate patient population and remember that only up to $20 \%$ of patients will have concomitant pulmonary tuberculosis.

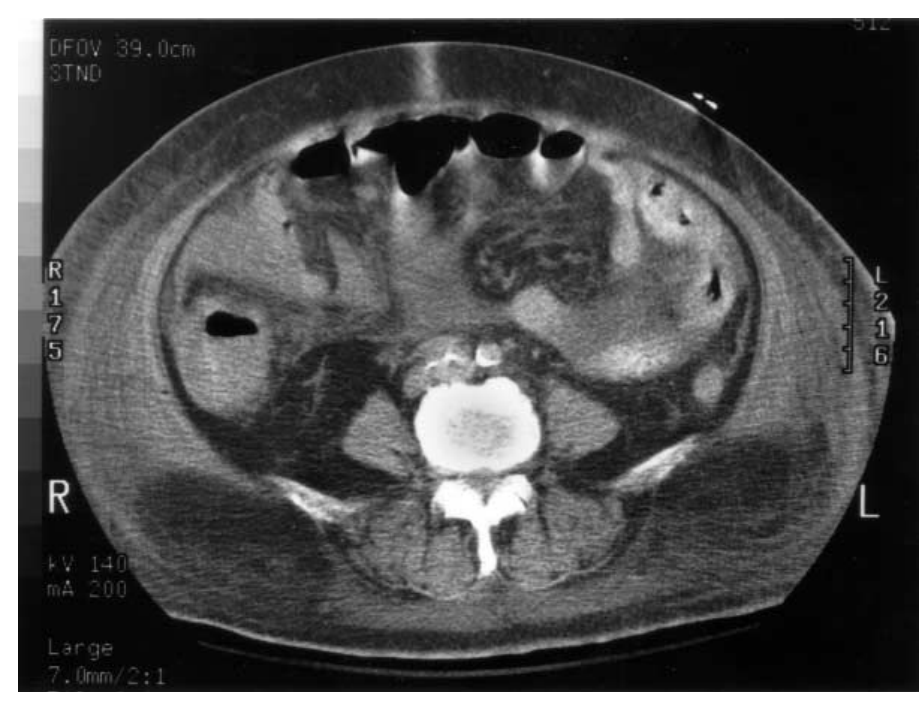

Figure 1. CT scan demonstrating chronic wall thickening involving the cecum and portion of the ascending colon, abdominal ascites, bilateral pleural effusions and diffuse bilateral ground glass opacities within the lung fields. 